\title{
Dimensiones virtuales de la ciudad: apropiación urbana mediada por el uso de la aplicación digital Pokémon GO
}

\begin{abstract}
Resumen
El presente artículo se deriva del proyecto macro del Grupo de Investigación Xisqua: "Nociones del territorio: ciudad, mapa e imaginario". El estudio que presenta es una reflexión acerca de las dimensiones virtuales de la ciudad como una oportunidad para el análisis de las relaciones urbanas que se gestan en el uso de aplicaciones móviles y la comprensión de la apropiación del territorio.
\end{abstract}

En este caso, se tomó como objeto de estudio a los usuarios de la aplicación Pokémon GO en la que se detecta la oportunidad de analizar la interacción espacial entre la realidad y la virtualidad en el contexto urbano.

El desarrollo del proyecto se plantea como un acercamiento piloto en el que se realizó un ejercicio de indagación a partir de técnicas como la observación participante y de instrumentos como la aplicación de encuestas a los usuarios del juego. Este acercamiento al tema, supone la posibilidad de establecer una nueva perspectiva para el abordaje del territorio y de las nociones que se gestan entre la interacción del ciudadano y el entorno urbano desde escenarios virtuales.

\author{
Carlos Mario Rodríguez R. \\ Magíster en Entornos Virtuales de \\ Aprendizaje \\ Docente asociado e investigador \\ Universidad de Boyacá \\ Boyacá, Colombia \\ Correo electrónico: carrodriguez@ \\ uniboyaca.edu.co \\ ๑ orcid.org/0000-0002-5670-7393 \\ Google Scholar
}

Héctor Fernando Martínez Martínez

Máster en Creación Digital

Docente asistente e investigador

Universidad de Boyacá

Boyacá, Colombia

Correo electrónico: phermartinez@

uniboyaca.edu.co

ำ orcid.org/0000-0001-5890-7869

Google Scholar

Recibido: Noviembre 1 de 2017

Aprobado: Octubre 5 de 2018

Palabras clave:

Ciudad virtual, dispositivos móviles, Pokémon GO, territorio. 


\section{Virtual dimensions of the city: Urban appropriation mediated by the use of the Pokémon GO digital application}

\begin{abstract}
This paper is derived from the Macro Project of the Xisqua Research Group: Notions of Territory: City, Maps and imaginart. The study presented reflects on the virtual dimensions of the city as an opportunity for analysis of urban relations that are developing in the use of mobile applications and the understanding of the appropriation of the territory.
\end{abstract}

In this case the users of the Pokémon Go application were taken as the object of study, in which the opportunity to analyze the spatial relationships between reality and virtuality in the urban context is detected.

The development of the project is proposed as a pilot approach in which an exercise of inquiry was carried out using techniques such as participant observation and instruments such as application of surveys to the users of the game. This approach to the subject suposses the possibility of establishing a new perspective for the approach to the territory and to the notions that are developing between the interaction to the citizen and the urban environment, in this case from virtual scenarios.
Key words:

Virtual city, mobile device, Pokémon Go, territory. 


\section{Introducción}

El desarrollo del proyecto macro "Nociones del territorio", del Grupo de Investigación Xisqua', ha implicado el acercamiento y comprensión de los fenómenos urbanos que se gestan entre la relación de los habitantes con el entorno. En ese sentido las miradas interdisciplinares de los integrantes del grupo han permitido configurar diferentes lecturas de la ciudad de Tunja ${ }^{2}$, lugar que ha sido tomado como principal escenario de análisis.

Hasta el momento, la reflexión se ha centrado en fenómenos sociales como los imaginarios urbanos y la apropiación del habitante por la ciudad. Esto ha hecho posible establecer lecturas urbanas desde el color, las percepciones sensoriales y las cartografías participativas entre otros. No obstante, el grupo no ha sido ajeno a los cambios y dinámicas que ha provocado el auge de la tecnología en los contextos urbanos y se ha planteado la posibilidad de aplicar las mismas metodologías de análisis en las nociones que se gestan en esta dimensión virtual del territorio. En este caso, esta dimensión no existe solamente en la mente o el imaginario de los habitantes sino que está mediada por un escenario virtual.

Inicialmente se ha propuesto el desarrollo de un ejercicio piloto, que permita identificar la pertinencia y posibilidad de análisis de la ciudad virtual. Con el objetivo de describir las nuevas dinámicas urbanas que se gestan en la dimensión virtual de la ciudad, se ha tomado como foco de estudio a los usuarios de la aplicación Pokémon GO. Este juego de realidad aumentada está basado en la popular serie japonesa de los 90 que, además de incluir la temática de capturar monstruos de diversas formas, tamaños y colores, se ha convertido en un fenómeno que ha permeado varias generaciones.

\footnotetext{
${ }^{1}$ Grupo de investigación de la Facultad de Arquitectura, Diseño y Urbanismo y el programa de Diseño Gráfico de la Universidad de Boyacá. El grupo desarrolla proyectos sobre la línea de "Diseño y sociedad". Ver trabajo en www.grupoxisqua.wordpress.com

${ }^{2}$ Tunja, ciudad intermedia del interior de Colombia, es la capital del departamento de Boyacá y se encuentra ubicada a 135 kilómetros de Bogotá; su extensión territorial es de 118 kilómetros cuadrados. Es una ciudad reconocida por el crecimiento y desarrollo que ha tenido en las últimas dos décadas y por ser un importante centro universitario y cultural del país.
} 
El juego plantea una relación directa entre el territorio físico y el virtual en la medida en que se basa en la geolocalización del territorio y la trama urbana, en el desplazamiento real por el espacio y la combinación de la realidad con la virtualidad a partir de las posibilidades tecnológicas de los dispositivos móviles de alta gama.

De esta manera, como se verá en los siguientes apartados, se desarrolló un proceso de indagación en el que se abordaron usuarios de la aplicación Pokémon GO en la ciudad de Tunja que aportaron información relacionada con los desplazamientos en la ciudad y las permanencias, recorridos y lugares. Todo ello permitió identificar nuevos significados y formas de relacionarse con el espacio público.

Así pues, se propone una lectura de otra dimensión de la ciudad; condicionada por las características de un entorno virtual que transforma el espacio físico y lo adapta a un escenario imaginado, pero tangible en los dispositivos móviles.

\section{Contextualización}

\section{¿Qué es Pokémon GO?}

Pokémon GO es un juego desarrollado por Niantic y Nintendo para dispositivos móviles con sistema operativo Android o iOS. Fue lanzado en julio de 2016 y durante el segundo semestre del mencionado año recibió una atención mediática que lo llevó a ser considerado como la aplicación-videojuego con mayor éxito inmediato en la historia, Ilegando a romper cinco récords Guinness ${ }^{3}$.

\footnotetext{
${ }^{3}$ De acuerdo al portal Universia, Pokémon GO superó cinco récords Guinness a un mes de su lanzamiento: mayores ingresos generados por un juego para teléfonos móviles durante su mes de lanzamiento; el juego con más descargas registradas en su primer mes a disposición del público; la App más popular para iOS y Android en más de 70 países de manera simultánea; el juego para móviles que generó los mayores ingresos de manera simultánea en varios países durante su mes de lanzamiento y primer juego en superar los 100 millones de dólares en ingresos netos con apenas 20 días en el mercado.
} 
El juego, está basado en la famosa serie de anime japonesa de los 90 del mismo nombre. La narrativa presenta un mundo ficticio lleno de criaturas llamadas pokémon que se asemejan a animales o plantas, pero tienen poderes y capacidades evolutivas. En este mundo, los niños más valientes se forman como "maestros pokémon"; lo cual implica realizar viajes por el mundo, cazar criaturas, entrenarlas y luchar en batallas para ganar medallas y torneos (Pokémon/Nintendo, 2017). De esta manera el juego no es un fenómeno comunicativo aislado sino que responde a una convergencia de lenguajes de medios (Scolari, 2013), siendo posible considerarlo como parte de una narrativa transmedia puesto que propicia que el seguidor del programa retome la historia que conoció en la televisión y continúe el relato desde su propia experiencia propiciando así una expansión del universo narrativo.

En gran parte, el éxito de Pokémon GO se debe a la articulación del mundo de ficción de Pokémon con los juegos de rol y los recursos de la tecnología móvil, especialmente la realidad aumentada. En ese sentido puede considerarse como un juego de rol PvE (jugador versus entorno) en el que la trama argumental describe un viaje por el mundo para capturar, coleccionar y entrenar los mencionados monstruos. Los personajes, son avatares creados y personalizados por los usuarios y cada jugador puede elegir ser parte de uno de los tres equipos existentes (Valor, Instinto o Sabiduría). No obstante, y a diferencia de juegos como Second Life y Warcraft, a la fecha no es posible la interacción PvP (jugador versus jugador). Es decir que el juego está diseñado para que el usuario se enfrente al entorno.

Una de las particularidades del juego consiste en que articula el ambiente real con un plano virtual. Vale la pena mencionar que la campaña publicitaria del juego señaló como beneficio del mismo, el propósito de hacer salir a la calle al jugador para socializar con otras personas y lugares; de esta manera romper con el sedentarismo que caracteriza la mayoría de videojuegos. Esto implica 
que el jugador se tiene que desplazar a diferentes lugares, a otras ciudades e incluso otros países para tener un buen desempeño en el juego (Infobae, 2017).

El diseño del juego implica un nivel alto de inmersión del jugador. Dawley y Dede (2014) mencionan que:

las tecnologías inmersivas, favorecen la posibilidad de experimentar a través de la acción activa del usuario, de forma que éste pueda a su vez relacionarse con los elementos formativos e igualmente con el resto de los elementos del entorno, volviéndose colaborativo y motivador. (p. 379)

De esta manera el escenario del juego es un plano del contexto urbano real enriquecido con elementos que refuerzan la trama y otorgan nuevos significados a lugares y espacios como parte de la experiencia de inmersión del jugador.

Es necesario explicar que una vez instalada la aplicación en el dispositivo móvil, la dinámica de juego consiste en caminar con el GPS activo para buscar estos monstruos. La captura de un pokémon requiere de ciertos elementos que se consiguen en las pokeparadas; que corresponden a hitos urbanos como restaurantes, monumentos o parques. Allí se obtienen pokebolas y otros objetos que facilitan el desempeño en el juego. Cabe destacar que tanto las pokeparadas como los gimnasios pokémon son distribuidos en el mapa de toda la ciudad y son situados en lugares emblemáticos y de referencia a los que generalmente el transeúnte puede acceder.

\section{Ecosistema digital de Pokémon GO}

Realidad aumentada, geolocalización y cartografías digitales

De acuerdo a los elementos mencionados anteriormente, la aplicación retoma la narrativa de la serie y es desarrollada a partir de un ecosistema digital que combina la experiencia del usuario con el mundo real —mediada por la 
tecnología- a través de la integración de dispositivos móviles con el uso de realidad aumentada (RA) y geolocalización.

La realidad aumentada es una tecnología en desarrollo que ha aprovechado las pantallas de los dispositivos móviles para ofrecer a los usuarios una visión mejorada del mundo real. En este caso, se sobrepone información digital sobre el entorno real. De acuerdo a Basogain et al. (2007) "la Realidad Aumentada es una tecnología que complementa la percepción e interacción con el mundo real y permite al usuario estar en un entorno real aumentado con información adicional generada por el ordenador" (p. 24). Esta tecnología se encuentra hoy extendida en todos los campos del saber, pues se caracteriza por ser muy versátil. Por su parte, el contexto urbano ha sido un escenario ideal para el desarrollo de la RA dado que el objetivo que propone esta tecnología implica el desplazamiento e interacción del usuario con el mundo real.

Día a día emergen nuevas aplicaciones y desaparecen otras, entre las iniciativas con mayor trayectoria se encuentran Wikitude o Ingress. El primer caso mencionado es una aplicación que existe desde 2008 y que con el paso del tiempo ha logrado incorporar la realidad aumentada a casi cualquier aspecto del entorno. Uno de los usos más frecuentes de la aplicación es el reconocimiento de la naturaleza de los lugares en el contexto urbano. Con base en ello los viajeros o residentes de la ciudad pueden recibir información de hospedaje, restaurantes y recomendaciones, al escanear con el dispositivo móvil el ambiente que los rodea.

Ingress — que es tal vez el antecedente más directo de Pokemón GO- es un juego bélico que, bajo el eslogan de "el mundo alrededor no es lo que parece", propone una narrativa futurista en la que la humanidad se debate entre aceptar o no el dominio alienígena. Los eventos del juego ocurren en espacios reales e implican el desplazamiento del usuario dentro de los espacios urbanos. 
En Pokémon GO, la RA es implementada justamente para sobreponer las imágenes de los pokémon sobre la visión de la cámara del móvil en el mundo real. De esta manera se retoma la idea de la búsqueda de monstruos en el mundo real y hace posible ver un Rattata o un Pidgey ${ }^{4}$ en la calle o en la oficina, o ver un gimnasio pokemón sobre la imagen de un reconocido edificio de la ciudad. Este componente ha sido quizá uno de los mayores atractivos del juego, por lo que se ha viralizado en redes sociales.
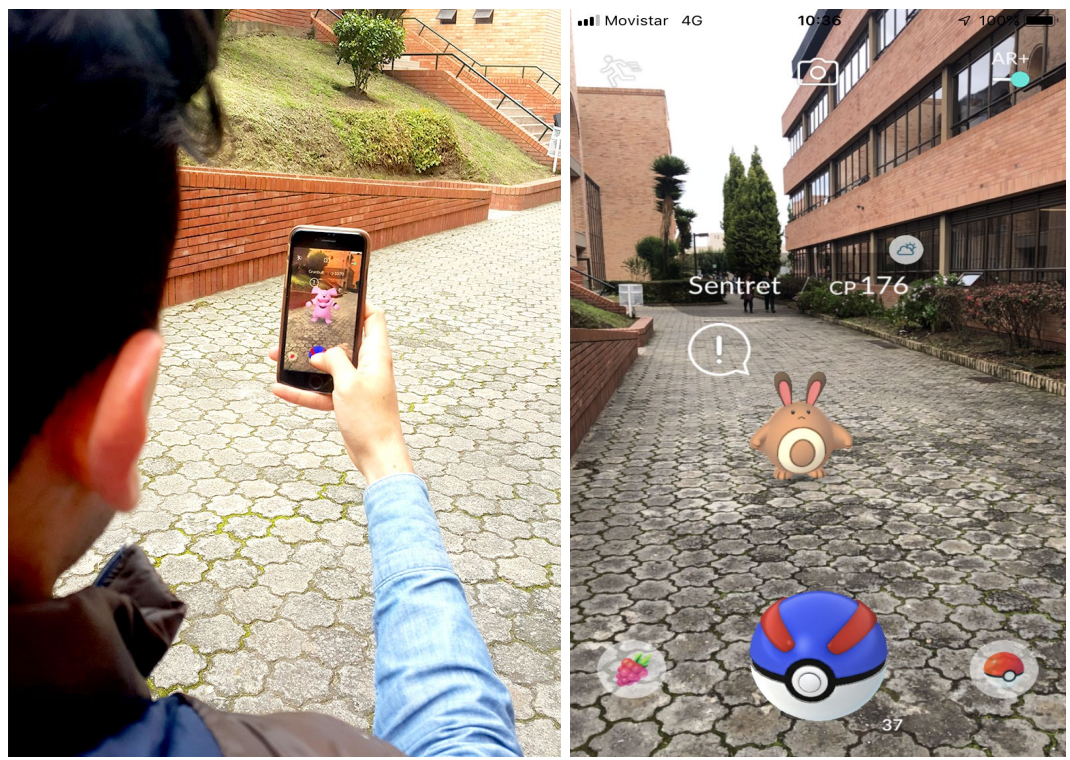

Figura 1. Ejemplo de realidad aumentada, sobreposición de personaje sobre la realidad. Fuente: elaboración propia por parte de los autores.

\footnotetext{
${ }^{4}$ Rattata y Pidgey son dos de las criaturas más populares de la serie. Como son semejantes a ratones y palomas, en el plano virtual de la ciudad es muy fácil encontrarlos en lugares como parques y plazas.
} 
Cuando se comenzó esta iniciativa investigativa, las primeras hipótesis identificaban a la RA como uno de los principales componentes para analizar la relación del habitante con la ciudad. Sin embargo la tecnología RA no es imprescindible dentro de la aplicación sino una característica que se puede descartar sin afectar el desempeño dentro del juego. De hecho, en el ejercicio de observación de los participantes, fue notable la cantidad de usuarios que tras perder el encanto de los primeros días de juego desactivaron la opción de RA y continúan sin esta característica. Si bien la RA constituye un acercamiento inicial entre la relación del jugador y el contexto urbano, es notable que esto se dé en gran medida por el uso de la geolocalización y los mapas digitales. Por esta razón, la investigación aborda el análisis de estos elementos.
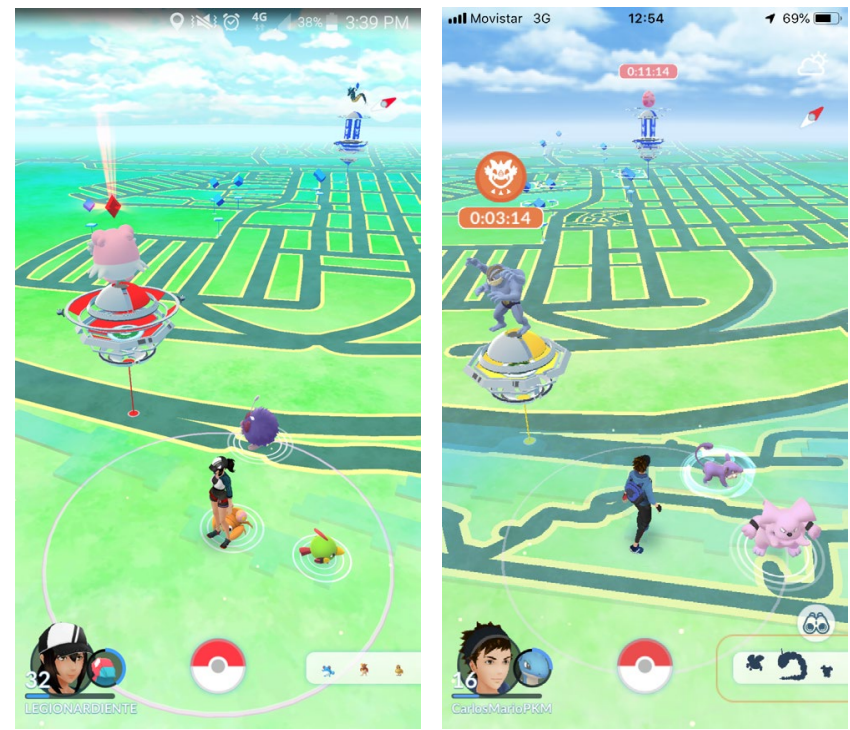

Figura 2. Ejemplos del jugador situado de manera virtual sobre el plano de la ciudad. Fuente: elaboración propia por parte de los autores. 
Un componente característico del juego es el hecho de que genera en el usuario la necesidad de desplazarse en el espacio físico real. La aplicación retoma la información de Google Maps y utiliza el GPS de los móviles para posicionar a los jugadores en el mapa. De esta manera se dibuja toda una nueva trama urbana digital que obliga al jugador a desplazarse si quiere avanzar en el juego.

Jugar al Pokemon Go es, principalmente, desplazarse en el espacio, que aparece estructurado en una serie de puntos de referencia semi arbitrarios identificados en el mapa del juego. El hecho de que no podamos saber a ciencia cierta en donde encontraremos a los Pokemones que deseamos nos invita a actuar como flâneurs a la espera de experiencias posibles, así como las Pokeparadas y Gimnasios nos incitan continuamente a desviarnos de las rutas establecidas por el pragmatismo de la vida cotidiana. (Vila y Vilar, 2016)

En la serie original, el mapa es un elemento clave; pues los protagonistas deben desplazarse por bosques, islas y regiones para buscar los pokémon y acceder a gimnasios y competencias. En la aplicación los hitos y nodos de las ciudades han sido reemplazados por elementos del juego que conservan los nombres reales e información gráfica y geográfica de los lugares, aunque les otorga un nuevo uso y significado. Es posible, por ejemplo, ver un monumento convertido en pokeparada o un edificio en gimnasio pokémon. Esto ha implicado una relación directa entre el jugador y el espacio público. El juego ha propiciado nuevas dinámicas urbanas, nuevos itinerarios y desplazamientos. Vila y Vilar (2016) explican:

\footnotetext{
la geografía específica del mundo Pokémon "clásico", empleada también en la serie de animé, viene a ser reemplazado por la construcción de nuestras ciudades humanas, atravesadas por siglos de historia social y natural, intereses inmobiliarios y políticas de Estado. Esto implica una interacción entre el espacio público, con todos sus condicionamientos socioecónomicos, y los elementos generados digitalmente para ser captados por el GPS.
}

Esta nueva cartografía digital y manera de entender el contexto ha llevado a la construcción de nuevas prácticas urbanas en los jugadores. En este ejercicio experimental se tomó como caso de estudio a la ciudad de Tunja, lugar en el que 
se revisó la interacción de los jugadores con la ciudad a partir del ecosistema digital: realidad aumentada, geolocalización y cartografías digitales.

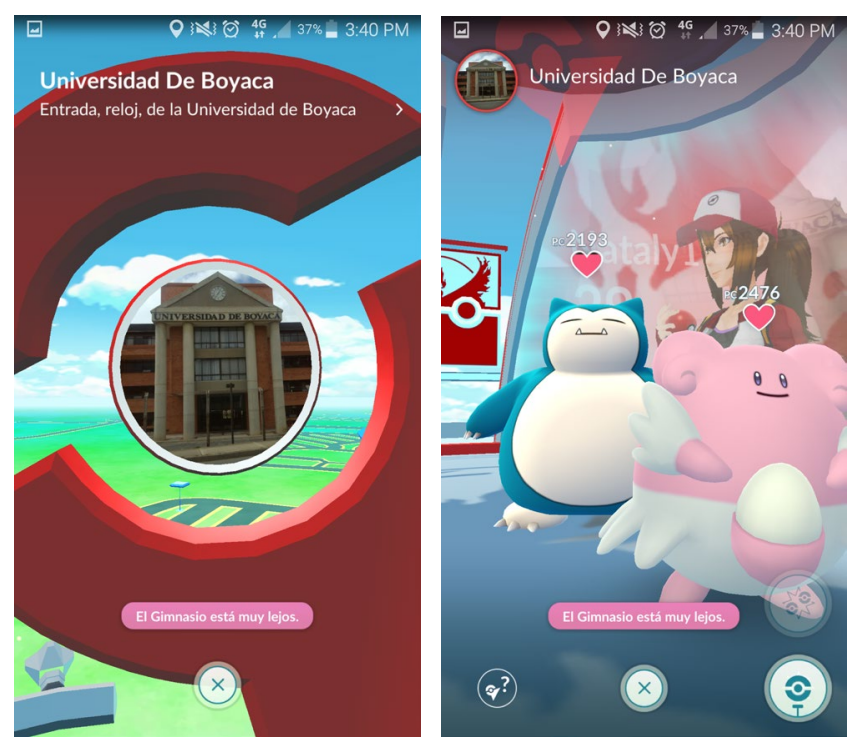

Figura 3. La Universidad de Boyacá, escenificada como gimnasio pokémon. Fuente: elaboración propia por parte de los autores.

\section{Materiales y métodos}

Bajo un enfoque cualitativo y un tipo de estudio descriptivo-fenomenológico, que tuvo como objetivo describir las nuevas dinámicas urbanas que se gestan en la dimensión virtual de la ciudad, se realizaron tres tipos de acercamiento al objeto de estudio y un ejercicio de inmersión y experiencia de campo con el juego. 
Las técnicas e instrumentos utilizados en este ejercicio experimental incluyeron una observación participante en la que los investigadores estuvieron inmersos en el juego y comprobaron a través de la experiencia la dinámica de los jugadores. En esta actividad se realizaron también entrevistas informales, se registró la información con apuntes en el diario de campo y fotografías.

Por otra parte, y aprovechando la red de jugadores establecida en grupos de chat, se realizó una encuesta virtual en la que los usuarios fueron cuestionados por aspectos como el nivel, avance y dinámica del juego; así como por la experiencia relacionada con la interacción entre el mundo real y el virtual.

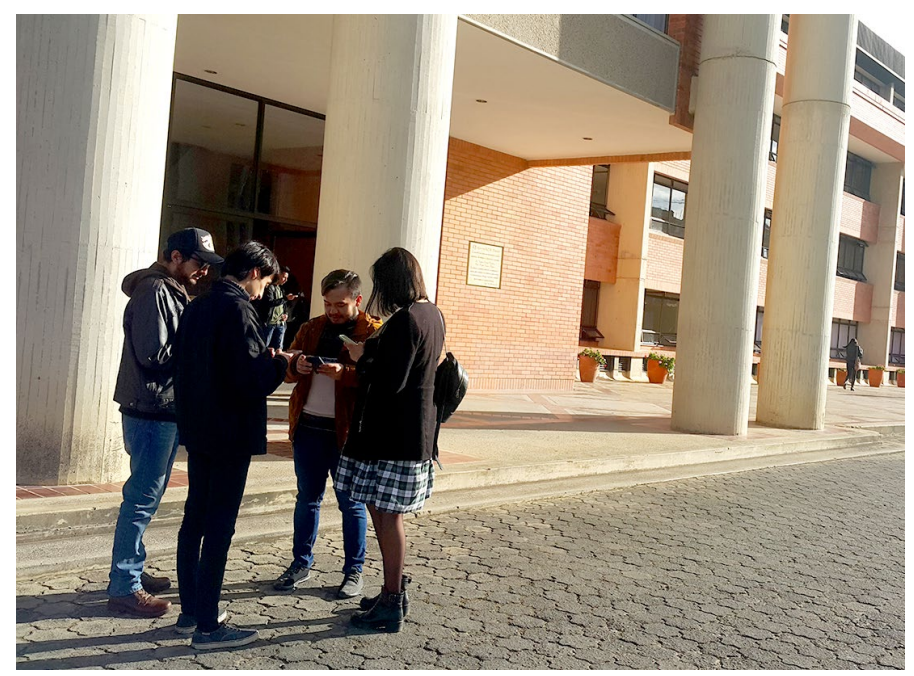

Figura 4. Jugadores reunidos en el Edificio Central de la Universidad de Boyacá, uno de los "gimnasio pokémon" más populares. Fuente: elaboración propia por parte de los autores. 
El primer ejercicio de campo consistió en la instalación de la aplicación y descubrimiento de primera mano de la metodología del juego. En este caso, los investigadores se dirigieron a sectores de la ciudad de Tunja como son: el barrio Los Muiscas, la Universidad Pedagógica y Tecnológica de Colombia (UPTC) y la Universidad de Boyacá. En este proceso se reconoció el potencial objeto de estudio, los jugadores y usuarios de la aplicación.

De la misma manera se identificaron aspectos como la existencia de tres equipos dentro del juego; y si bien a la fecha la aplicación no permite la interacción directa entre los jugadores, estos han establecido redes de comunicación alternas como grupos de WhatsApp y Facebook. De esta forma se aportan consejos y trucos continuamente, así como los lugares preferidos para la reunión de los jugadores. También se establecen puntos de encuentro, estrategias para apoderarse de los gimnasios o caminatas para cazar pokémon.

La experiencia inmersiva incluyó varias caminatas y recorridos. Esto hizo posible comprender el juego, desplazarse por lugares y establecer un mapeo inicial de usuarios a entrevistar. La observación directa permitió identificar este primer contacto, conversar con los jugadores y recolectar los primeros datos acerca de las relaciones espaciales y sociales que se comenzaban a gestar entre la comunidad de Pokémon GO.

En Buenos Aires se desarrolló un proyecto de naturaleza similar que incluyó también un ejercicio de observación directa. Los resultados similares al ejercicio desarrollado en Tunja dan muestra de que, si bien el juego propone unas dinámicas específicas, el conocimiento del contexto que tiene el usuario potencia otro tipo de actividades e interacción con el entorno tal como lo explica Ibarrola (2017): 
esta salida al campo resultó interesante porque dio cuenta de una serie de relaciones sociales y espaciales que se despliegan a través de este binomio juego/tecnología que es Pokémon Go.

Los jugadores hacen uso de todo su conocimiento del entorno para jugar este juego. Mi experiencia junto a un entrenador da cuenta del manejo del espacio local de una manera muy precisa, para encontrar las paradas, de una memoria visual importante, para poder perseguir a los Pokémon que se escapan, etc. [...] Mi acompañante comienza a desplazarse hacia zonas que no suele frecuentar. Las áreas del barrio, frecuentadas por sectores de la población más "pudientes", comienzan a ser una región de tránsito frecuente. Cuando Manuel me dice que viene caminando a mi casa ahora, me está diciendo que la tecnología está modificando su itinerario cotidiano. En este sentido se podría decir que Pokémon Go está, de alguna forma, reconfigurando el espacio social de los sujetos que participan del juego. (p. 8)

La experiencia junto a los jugadores nos demostró que nuevos imaginarios urbanos se han gestado en medio de las dinámicas del juego, los lugares han adquirido nuevos significados y los jugadores han adaptado una nueva manera de reconocer el contexto urbano.

Una vez superado este primer ejercicio, se tomó como muestra de análisis a 50 jugadores de Pokémon GO y a los mismos se les aplicó una encuesta digital conformada por 12 preguntas abiertas y cerradas que indagó por las relaciones del espacio físico con el espacio virtual en la ciudad. Las respuestas fueron sistematizadas y analizadas a partir de la elaboración de mapas, zonas, sendas e hitos para caracterizar esta ciudad virtual.

Una de las hipótesis que se plantearon al comienzo del ejercicio investigativo estuvo enmarcada en la pertinencia de analizar esta nueva dimensión de la ciudad desde los enunciados de Lynch (1984), que hasta el momento habían sido pertinentes para comprender a la ciudad de los imaginarios; sin embargo se cuestionó si las categorías propuestas aún eran válidas para entender la dinámica urbana de hoy. De esta manera se retomaron los enunciados y se establecieron las categorías, lo cual nuevamente constituyó un ejercicio experimental en el que la triangulación de la información fue demostrando la 
efectividad de los lineamientos de la imagen de la ciudad de Kevin Lynch; tal como se verá en los siguientes apartados.

\section{Primeras caracterizaciones de la población abordada}

Una vez realizado el proceso de observación participativa, y contacto directo con la aplicación, el proceso experimental hizo posible identificar una población de usuarios a quienes por medio de comunidades de jugadores organizadas en WhatsApp fue posible aplicar un cuestionario online del que se desprendieron las primeras caracterizaciones de la ciudad.

El rango de edad más frecuente entre los usuarios encuestados fue de la generación nacida en los 90, es decir usuarios que crecieron con la serie y que previamente ya estaban contextualizados con la trama argumental del juego.

En estas comunidades, el nivel de desempeño en el juego es en su mayoría de 16 a 25 puntos. En la experiencia particular de los investigadores se constató que subir de nivel y ganar puntos es una tarea que requiere tiempo, es decir que si se compara el nivel de los jugadores encuestados con la fecha de lanzamiento del juego y la fecha de aplicación de la encuesta se demuestra una dedicación significativa al mismo; calculada en 2 a 4 horas al día.

Es decir que estas personas ya tienen una gran experiencia en el uso de la aplicación, lo cual es evidente en el hecho de que la mayoría de los usuarios ha recorrido entre 30 y 60 kilómetros jugando en la ciudad. Es importante mencionar que la encuesta fue aplicada en septiembre de 2016, a pocos meses del lanzamiento del juego. 

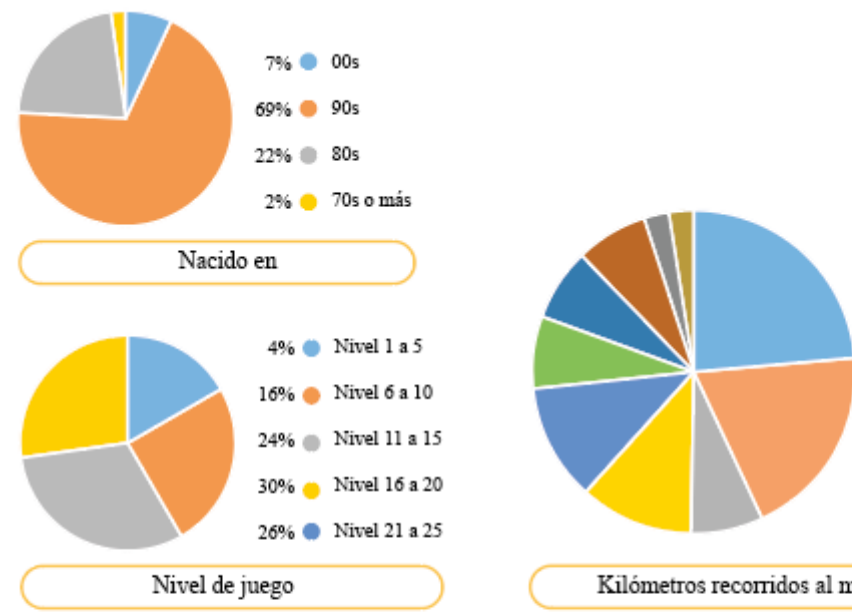

$24 \%$ Entre $0 \mathrm{~km}$ a $30 \mathrm{~km}$ $19 \%$ Entre $31 \mathrm{~km}$ a $60 \mathrm{~km}$ $7 \%$ Entre $61 \mathrm{~km}$ a $90 \mathrm{~km}$ $12 \%$ Entre $91 \mathrm{~km}$ a $120 \mathrm{~km}$ $12 \%$ Entre $121 \mathrm{~km}$ a $150 \mathrm{~km}$ $7 \%$ Entre $151 \mathrm{~km}$ a $180 \mathrm{~km}$ $7 \%$ Entre $181 \mathrm{~km}$ a $210 \mathrm{~km}$ $7 \%$ Entre $211 \mathrm{~km}$ a $240 \mathrm{~km}$ $3 \%$ Entre $241 \mathrm{~km}$ a $270 \mathrm{~km}$ $2 \%$ más de 270

Kilómetros recorridos al mes usando la aplicación

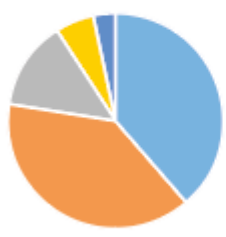

$40 \%$ - 1 bora o menos al día

$39 \%$ - 2 a 4 horas al dia

$14 \% \bigcirc 4$ a 6 horas al dia

$5 \%$ a 6 horas al dia

$2 \%$ Más de 8 horas

Frecuencia de uso de la app Pokémon Go

Figura 5. Primera caracterización de los usuarios abordados en el proyecto piloto. Fuente: elaboración propia por parte de los autores.

Por otra parte, se identificaron las zonas más frecuentes para el juego: los usuarios encuestados revelaron que la UPTC es el lugar que reúne las mayores condiciones para la realización de recorridos. Asimismo, el barrio Los Muiscas ubicado al norte de Tunja y el centro de la ciudad son lugares también propicios para el juego. 
Así, fue posible identificar imaginarios urbanos relacionados con el peligro y la seguridad. En el ejercicio fue frecuente escuchar comentarios sobre noticias de robos a personas que utilizan el juego, razón por la cual los usuarios han establecido zonas seguras para caminar y jugar. En Tunja, zonas como el barrio El Bosque y el Terminal de Transportes fueron asociados por los jugadores como espacios no seguros donde no es recomendable jugar.

Finalmente se indagó sobre las razones y motivos principales para utilizar el juego. En este caso, las respuestas con mayores resultados tuvieron que ver con sentimientos de nostalgia por la infancia y con ver realizado por medio de la tecnología lo que en tiempo atrás solo era parte de la ficción.
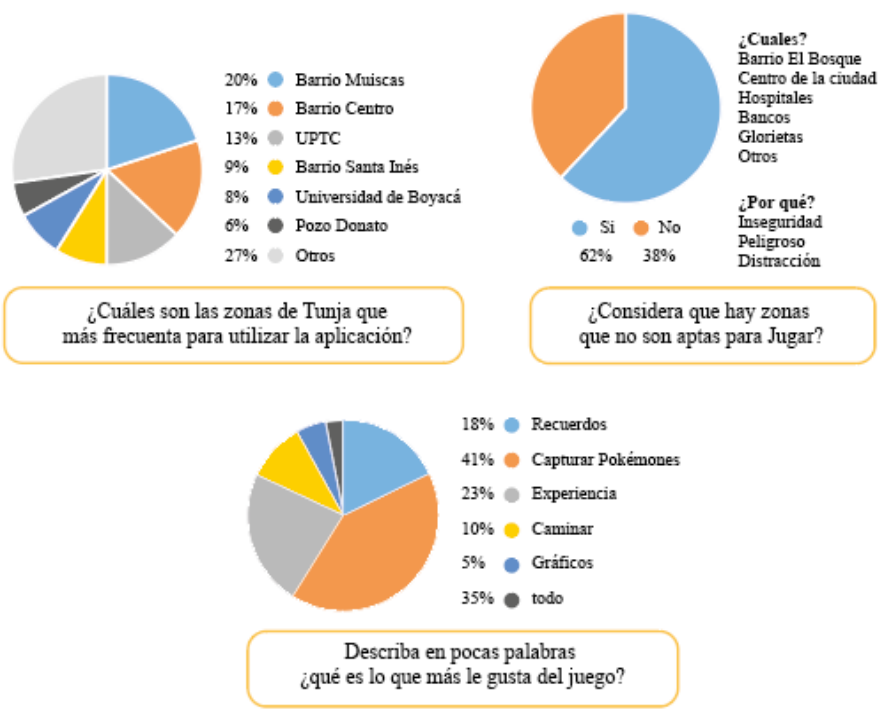

Figura 6. Caracterización de las zonas de juego y percepción de los usuarios. Fuente: elaboración propia por parte de los autores. 


\section{Resultados de la indagación experimental}

Una vez caracterizada la población, que resultó ser en su mayoría estudiantes de pregrado de las universidades de la ciudad de Tunja, se procedió a indagar por las relaciones que estas personas establecen con la trama urbana mediada por la aplicación de Pokémon GO y siguiendo los enunciados de Lynch (1984). Este ha sido, como se mencionó anteriormente, el mismo procedimiento utilizado para el desarrollo de los análisis previos del Grupo de Investigación Xisqua.

\section{Los nodos de la ciudad}

Los nodos son aquellos puntos estratégicos que funcionan como focos intensivos en los que se concentra un gran flujo de personas. Generalmente son empleados como referencias mentales para la ubicación dentro de la ciudad y se constituyen como el punto de partida de las sendas (Rodríguez, 2014). En este caso, los nodos han sido comparados con las pokeparadas dado que dentro de la trama argumental del juego cumplen con estas funciones.

En los resultados de la indagación los gamers identificaron en la ciudad de Tunja lugares como la iglesia del barrio Los Muiscas, la UPTC, El Pozo de Donato y la Plaza de Bolívar como pokeparadas o nodos dentro del mapa del juego. Algo para resaltar es cómo estos lugares han sido testigos de cambios en las dinámicas urbanas de esta comunidad, pues debido al juego son ahora lugares de reunión y paso obligatorio de jugadores. Espacios que antes no recibían atención del transeúnte, son ahora sitios de encuentro y reunión. 
Rodríguez, C.M. y Martínez, H.F. / Dimensiones virtuales de la ciudad: apropiación urbana mediada

por el uso de la aplicación digital Pokémon GO

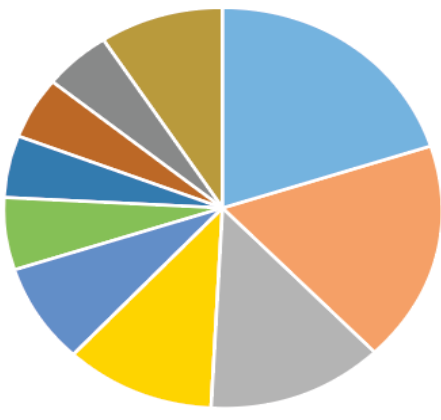

$20 \%$

$18 \%$

$13 \%$

$11 \%$ Plaza de Bolivar - monumento

$8 \%$ Despacho Parroquial Muiscas

$6 \%$ Universidad Santo Tomás

$5 \%$ Barrio Suamox

$5 \%$ Mural Duende Irlandés

$5 \%$ Barrio Santa Inés

$9 \%$ Otros

Lugares más recordados constituidos como paradas pokémon

Figura 7. Nodos identificados dentro de la dimensión virtual del juego.

Fuente: elaboración propia por parte de los autores.

Los hitos de la ciudad

Los hitos o mojones, son elementos de gran relevancia dentro de la trama urbana; pues comúnmente se emplean como referentes para la ubicación dentro de la ciudad. Estos son elementos físicos que gozan de fama entre los ciudadanos como un edificio emblemático, una estatua, o un almacén (Rodríguez, 2014).

En la comparación con la ciudad planteada por el juego, los hitos corresponden a los gimnasios pokémon; pues son los referentes más relevantes del mapa y constituyen uno de los objetivos más importantes del juego: competir en un gimnasio. Estos "nuevos hitos" le han otorgado nuevos significados a los lugares de la ciudad y en la narrativa del juego se han establecido como escenarios de lucha y conquista. 
Los ciudadanos encuestados, señalaron que los principales gimnasios pokémon o hitos de la ciudad virtual son la Universidad de Boyacá y la UPTC. Además se destacaron lugares como el Terminal de Transportes, la glorieta del monumento a la Raza y el concesionario Toyota.

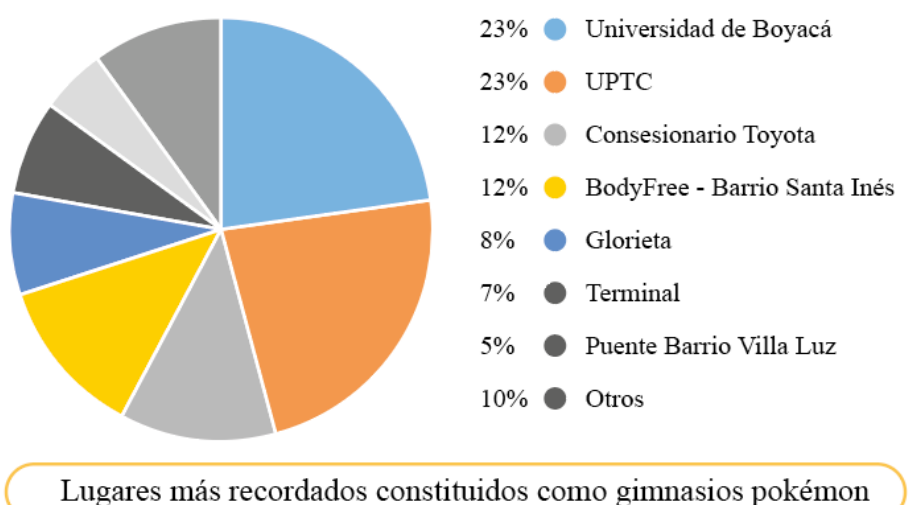

Figura 8. Hitos identificados dentro de la dimensión virtual del juego.

Fuente: elaboración propia por parte de los autores.

Los barrios de la ciudad

Los barrios son aquellas secciones de la ciudad con las que el observador asocia a un sector de la misma y a un grupo de personas. Son lugares en los que el ciudadano ingresa y hace recorridos (Rodríguez, 2014). Si bien la aplicación no propone una zonificación como tal, se decidió realizar un análisis de zonas o barrios debido a que durante la indagación se preguntó a los ciudadanos si consideraban que existían zonas de la ciudad que eran mejor para jugar que otras. 
Las respuestas mostraron que la mayoría sí opta por algunas zonas sobre otras; nuevamente se entrelazan la realidad con la virtualidad, ya que las razones de preferencia obedecen a imaginarios relacionados con sectores seguros o peligrosos. Un ejemplo de ello es que en investigaciones previas, lugares como el Terminal de Transporte recibió marcaciones relacionadas con la inseguridad y por lo tanto en este nuevo acercamiento la población reconoció al sector como una zona no segura para jugar.

Los gamers encuestados señalaron que los barrios o zonas predilectas para jugar son el barrio Los Muiscas, el centro de la ciudad, el barrio Santa Inés y el sector de la UPTC.

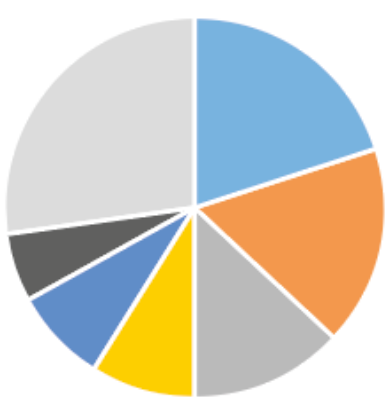
$20 \%$ Barrio Muiscas
$17 \%$ Barrio Centro
$13 \% \bigcirc$ UPTC
$9 \%$ Barrio Santa Inés
8\% Universidad de Boyacá
6\% Pozo Donato
$27 \%$ Otros

¿Cuáles son las zonas de Tunja que más frecuenta para utilizar la aplicación?

Figura 9. Barrios identificados dentro de la dimensión virtual del juego. Fuente: elaboración propia por parte de los autores. 
Las sendas de la ciudad

Las sendas son aquellas rutas y caminos que el ciudadano transita cotidianamente. Como se ha mencionado, uno de los elementos característicos de Pokémon GO es el hecho de que para tener éxito en el juego es necesario desplazarse puesto que este ofrece recompensas por los kilómetros andados y la búsqueda de pokémon implica ir de un lugar a otro.

En este caso, ha sido claro cómo el juego ha propiciado nuevas rutinas y lugares transitados. Una de las preguntas de la encuesta trataba sobre la posibilidad de que el jugador hubiese conocido nuevos lugares de la ciudad desde que utilizaba la aplicación. Un 42 \% respondió que sí, señalando que el juego hizo que conocieran lugares como iglesias o barrios alejados del centro de la ciudad o de los lugares que transitan normalmente.

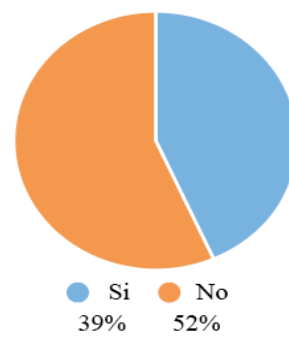

¿Desde que está utilizando la app ha visitado y/o conocido nuevos lugares de la ciudad? 
Así pues, los resultados mostraron que las sendas que surgieron a partir del juego en la ciudad de Tunja corresponden a lugares y sitios que los usuarios acostumbran a transitar para mejorar el desempeño del juego. Y están constituidas por la conexión entre nodos e hitos que, para el caso, vienen siendo gimnasios pokémon y pokeparadas. En el tiempo de observación e indagación, con los usuarios, se identificó que los jugadores las recorren varias veces al día; incluso, algunos realizan los recorridos en bicicleta. Las sendas que se caracterizaron son las siguientes:

1. Senda barrio Los Muiscas: esta senda con cinco paradas realiza un recorrido periférico por el barrio Los Muiscas, que está ubicado en el norte de la ciudad y muy cerca al sector universitario. Allí el recorrido implica el paso por nodos como el parque y la iglesia del barrio y por hitos como "la cruz de Los Muiscas" y las esquinas más comerciales. Es utilizada especialmente por los estudiantes de la Universidad de Boyacá.

2. Senda UPTC-Santa Inés: esta senda se caracteriza por ser un recorrido que parte de la UPTC; al interior de la universidad se establecen veintitrés paradas, lo que la constituye en el territorio con más adaptaciones al juego dentro de la ciudad. De allí se adentra al barrio más cercano, Santa Inés, con nueve paradas más. Vale la pena resaltar que la mayoría de los hitos se caracterizan por ser grafitis y murales, bastante evidentes en el sector y en las paredes de la institución. El recorrido también es un tour o recorrido turístico por estas manifestaciones visuales.

3. Senda centro: en el centro de la ciudad de Tunja, la senda que los jugadores han establecido perfectamente podría homologarse con un recorrido turístico por los principales sitios patrimoniales del Centro Histórico. La ruta parte desde la Plazoleta de Las Nieves y llega hasta la Iglesia de San Francisco. Allí establece un recorrido circular que rodea las cuadras aledañas a la Plaza 
de Bolívar. Entre los sitios destacables de la senda se encuentran: la Casa del Fundador, la Casa del Escribano, la Pila del Mono, monumentos, fuentes e iglesias.

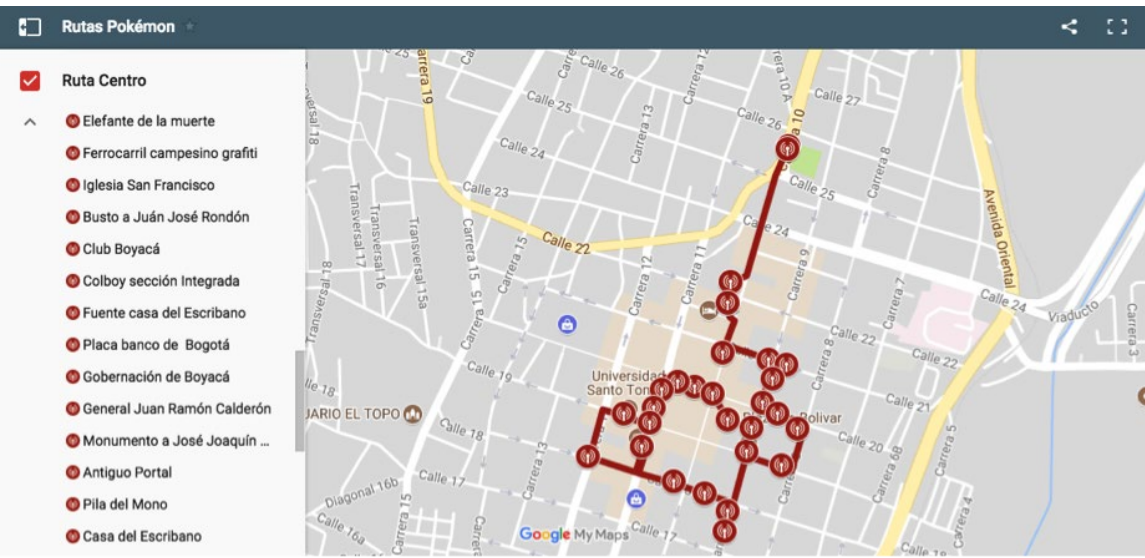

Figura 11. Mapa de sendas y rutas que se han gestado en la ciudad a partir del uso de la aplicación Pokémon GO. Fuente: elaboración propia por parte de los autores.

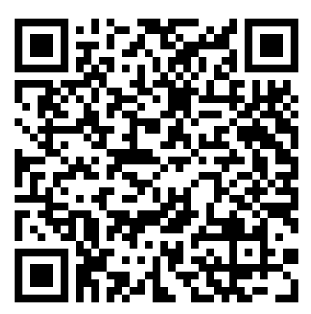

Figura 12. Con este código QR es posible acceder a un mapa interactivo con información de cada lugar. Fuente: elaboración propia por parte de los autores. 


\section{Discusión y conclusiones}

\section{Una dimensión virtual de la ciudad}

Este proyecto permitió el desarrollo de un proceso investigativo experimental que hizo posible establecer una serie de conclusiones y considerar estudios futuros a partir de estos resultados.

Con relación a los juegos y los mundos ficticios que los mismos plantean se abre el camino a todo un nuevo campo de investigación, que bien puede fundamentarse en las relaciones espaciales que establece el individuo con el mundo real y compararlas con la virtualidad. De acuerdo a Morales (2014): "los juegos se desarrollan en una realidad secundaria separada de la vida cotidiana y se rigen por un código especial que se hace visible ante el jugador mediante una representación simbólica" (p. 171). En ese sentido es relevante mencionar la articulación entre mundo real y mundo virtual que ha logrado Pokémon GO a través de tecnologías como la RA y la geolocalización.

Esto puede revaluar las teorías que hoy siguen siendo referenciadas, pues:

\footnotetext{
Johan Huizinga (1938) se refirió a finales de los años treinta en su libro Homo Ludens al "círculo mágico del juego" como un reducto de excepcionalidad que supone una separación espacio temporal entre la actividad de jugar y la vida cotidiana, y en la cual el jugador se sumerge de manera voluntaria aceptando comportarse según las reglas del mismo. (Morales, 2014, p. 171)
}

En ese mismo orden de ideas, es posible concluir que las barreras que separan la virtualidad del juego de la vida real son cada vez más difusas y que en estos nuevos mundos ficticios el individuo debe responder tanto a las reglas del juego como a las reglas del mundo real.

El encuentro entre las personas y los bits que pronosticaba Nicholas Negroponte, en 1995, es más que una realidad. La descripción prospectiva que el autor 
realizó en su libro Ser digital en la que imaginaba a una familia observando un partido de fútbol sobre la mesa de la sala con diminutos jugadores virtuales que lanzaban la pelota de un lado a otro, lejos de ser ficción en estos días, es algo que cualquier aplicación de RA podría resolver. Quizá, lo que no pronosticó el autor es que estas tecnologías podrían cambiar la manera en la que nos relacionamos con el entorno; y que tendrían gran incidencia en la imagen mental del contexto urbano y mediarían nuestras relaciones con la ciudad.

Estos nuevos fenómenos sociales han revelado también sistemas de comunicación en comunidad, representados por grupos de chat en la red social WhatsApp, en los que la comunicación es impersonal pero todos los individuos participan activamente puesto que siguen objetivos similares. A pesar de que el juego no permite la interacción entre usuarios, esta se da en otro escenario virtual que trasciende la aplicación y conforma una comunidad de usuarios. Pokémon GO ha propiciado la conformación de una red en la que cada usurario participante aporta y enriquece en menor o mayor medida el escenario virtual. Banús (2006) define el trabajo en red como "el resultado de la adopción de formas flexibles y participativas de organización, implementadas a la hora de crear y aplicar los conocimientos a la solución de problemas" (p. 5). Así que el intercambio de información, datos, localizaciones y estrategias sucede en muchos casos entre desconocidos que comparten un mismo contexto urbano y virtual y que buscan cumplir un mismo objetivo.

Si bien el escenario seleccionado para el desarrollo de la investigación es una ciudad intermedia de Colombia, ha sido posible analizar el fenómeno y comprobar cómo el contexto global ha impactado directamente al contexto local. En palabras de Borja y Castells (2000): "basándose en la nueva infraestructura tecnológica, el proceso de globalización de la economía y comunicación ha cambiado nuestras formas de producir, consumir, gestionar, informar y pensar" (p. 21). En ese sentido el mismo fenómeno ocurrido en 
escenarios tan grandes como Nueva York o Sídney, fue constatado en Tunja; no obstante, las particularidades del contexto local han hecho que el ciudadano adapte la plataforma a su propia realidad. La sociedad del conocimiento es un concepto de trasformación de la sociedad moderna asociado con las redes tecnológicas y las nuevas formas de comunicar; la UNESCO (2017) define que: "el acceso a la información es esencial como una precondición para el desarrollo de las Sociedades del Conocimiento" (p. 31). Por ello es posible ejemplificar una aproximación a la sociedad del conocimiento desde el fenómeno que ha producido el uso de este juego en la medida en que ha generado redes, ha cambiado los significados de los espacios y ha vinculado al usuario con la apropiación del entorno a través del uso de la tecnología.

Es evidente que el fenómeno comunicativo que produce la mediación de la tecnología ha permeado contextos culturales, sociales y urbanos y que ello ocurre ya en nuestros barrios y calles; surgen entonces nuevas hipótesis relacionadas con la adaptación de nuestras ciudades ante este panorama virtual, ¿debería existir una planeación urbana que considere también esta nueva apropiación de la ciudad?, ¿qué tan lejos está el desarrollo real del territorio frente al acelerado desarrollo del territorio virtual?

Por otra parte queda abierta la discusión que propone el surgimiento de imaginarios urbanos, ahora desde contextos virtuales. Recorrer la ciudad y reconocerla es una actividad que ya no está mediada solamente por las percepciones sensoriales y las construcciones colectivas sino que puede estar intercedida por dispositivos, plataformas virtuales y pantallas. Pokémon GO ha tejido toda una nueva capa de condiciones urbanas sobre la ciudad de Tunja con significados particulares, nuevas asociaciones a los lugares y nuevos recorridos e itinerarios. Ejemplo de esto, es que el juego ha hecho que los usuarios conozcan nuevos lugares dentro de la ciudad y se dirijan a zonas por las que usualmente no transitaban. Así pues, es un tema cuya exploración 
puede continuar y abre el panorama de nuevos estudios sobre aplicaciones como Waze o Google Maps.

La experiencia de este ejercicio reveló una nueva dimensión de la ciudad electrónica y virtual, al igual que nuevos imaginarios. Armando Silva (2016) la llama ciudad de aire porque "se parece mucho la ciudad imaginada a la digital, solo que la primera es mental y la otra, del aire, pero ambas son etéreas y avanzan de la mano. El mundo imaginado del futuro es tecnológico". Basta ser consciente de la manera en la que nos desplazamos dentro de una ciudad desconocida: ya no acudimos a indicaciones sino a mapas virtuales, a una voz electrónica que guía el camino; ya no es necesario memorizar los desplazamientos o ser poseedores de un gran conocimiento del entorno urbano; las ciudades ahora están contenidas en diferentes capas virtuales, y esto ha cambiado en definitiva la manera de relacionarse con la urbe. Sería posible plantear entonces la ciudad de Facebook, de Waze, de WhatsApp, de Twitter o de Google Street View; cada una con sus connotaciones y significados particulares.

\section{Referencias}

Banús, E.M. (2006). La estrategia de redes de conocimiento adoptada por UNESCO. En M. Albornoz y C. Alfaraz, Redes de conocimiento: construcción, dinámica y gestión (pp. 9-14). Buenos Aires, Argentina: RICYT.

Basogain, X. et al. (2010). Realidad aumentada en la educación: una tecnología emergente. Recuperado de http://files.mediaticos.webnode.es/200000016a645ea73b3/realidad\%20A..pdf.

Borja, J. y Castells, M. (2000). Local y global. La gestión de las ciudades en la era de la información. Ciudad de México, México: Taurus. 
Dawley, L. and Dede, C. (2014). Situated learning in virtual worlds and inmersive simulations. En J.M. Spector et al. (Eds.), The Handbook of Research for Educational Communications and Technology. New York, USA: Springer.

Ibarrola, D. (2017). ¡Atrápalos ya! Prácticas y relaciones sociales en torno a Pokémon Go. Lúdicamente, 6 (11).

Lynch, K. (1984). La imagen de ciudad. Barcelona, España: Gustavo Gilli.

Morales, J. (2014). Innovación en el diseño de los mundos ficticios en los videojuegos educativos. Un cambio hacia entornos más abiertos a la participación significativa y el aprendizaje. Revista KEPES, 11 (10), 167193.

Negroponte, N. (1995). Ser digital. Buenos Aires, Argentina: Editorial Atlántida.

Rodríguez, C.M. (2014). Imaginarios y cartografías urbanas: la ciudad de Tunja como caso de estudio. IconoFacto, 10, (14), 35-50.

Scolari, C. (2013). Narrativas transmedia. Cuando todos los medios cuentan. Barcelona, España: Deusto.

Silva, A. (07 de agosto de 2016). Ciudad de aire. El Tiempo.

UNESCO. (2017). Las piedras angulares para la promoción de sociedades del conocimiento inclusivas. Paris, Francia: UNESCO.

Vila, E. y Vilar, M. (2016). Perseguimos quimeras con un celular. Un examen del ficcional del Pokemon Go. Revista Luthor, 7 (29).

Como citar: Rodríguez, C.M. y Martínez, H.F. (2019). Dimensiones virtuales de la ciudad: apropiación urbana mediada por el uso de la aplicación digital Pokémon GO. Revista KEPES, 16 (19), 317-345. DOI: 10.17151/kepes.2019.16.19.13 